\title{
Militantisme et répression
}

\section{Daniela Cuadros et Daniella Rocha}

\section{(2) OpenEdition \\ Journals}

Édition électronique

URL : http://journals.openedition.org/conflits/18626

DOI : 10.4000/conflits. 18626

ISSN : 1777-5345

Éditeur :

CECLS - Centre d'études sur les conflits - Liberté et sécurité, L'Harmattan

Édition imprimée

Date de publication : 15 juin 2013

Pagination : 7-11

ISBN : 978-2-343-01116-5

ISSN : 1157-996X

Référence électronique

Daniela Cuadros et Daniella Rocha, « Militantisme et répression», Cultures \& Conflits [En ligne], 89 |

printemps 2013, mis en ligne le 15 juin 2013, consulté le 31 mars 2021. URL : http://

journals.openedition.org/conflits/18626 ; DOI : https://doi.org/10.4000/conflits.18626 


\title{
Militantisme et répression
}

\author{
Introduction
}

\section{Daniela CUADROS, Daniella ROCHA}

Daniela Cuadros est politiste. Ses travaux portent sur les mutations de l'engagement partisan après les transitions démocratiques. Rattachée au Centre européen de sociologie et de science politique (CESSP - UMR 8209), sa thèse doctorale en cours à l'Université de Paris 1 est consacrée à l'étude du militantisme communiste au Chili depuis les années 1980.

Daniella Rocha est professeure à l'Institut de Science Politique de l'Université de Brasilia et chercheuse associée à l'IRIS-EHESS. Elle a soutenu en 2007 une thèse sur les effets paradoxaux de l'ascension au pouvoir au sein du Parti des Travailleurs brésilien. Ses recherches actuelles portent sur les reconfigurations du militantisme partisan, ses processus de diversification et de professionnalisation, ainsi que sur les modalités nouvelles de production des élites politiques locales, en perspective comparée.

Consacré aux rapports entre militantisme et répression, ce dossier théma$\checkmark$ tique invite à étudier les transformations des pratiques et des savoir-faire militants dans les contextes répressifs, à travers l'étude des histoires de vie et des milieux militants. Il s'agit de prêter une attention particulière aux dynamiques d'adaptation et de reconversion de l'engagement politique compris comme un continuum au prisme de l'avant, du pendant et de l'après répression.

Depuis trente ans, la littérature sur la répression fait état des effets réciproques entre violence d'État et contestation 1. Dans les années 1990, la littérature se penche plus directement sur le binôme répression-contestation, en particulier au regard des conditions de possibilité de la répression, mais ses effets dissuasifs font aussi l'objet de nombreux travaux ${ }^{2}$. Divers auteurs

1. Voir par exemple le travail de Opp K., Roehl W., "Repression, Micromobilization, and Political Protest”, Social Forces, vol. 69, n² 2, 1990, pp. 521-547 et Della Porta D., Social Movements, Political Violence and the State. A Comparative Analysis of Italy and Germany, New York, Cambridge University Press, 1995. 
constatent ainsi la diversification des organisations d'opposition, l'entrée en politique de nouvelles couches de la population et le renforcement de réseaux militants préexistants ${ }^{3}$. Aussi est-il largement admis de penser que la répression participe à la création de conditions favorables à la contestation ${ }^{4}$. Au cours des années 2000, l'étude des interactions entre les autorités étatiques et les acteurs contestataires a suscité l'attention des chercheurs. De nouvelles perspectives de recherche sur les liens entre contestation et répression ont vu le jour aux États-Unis ${ }^{5}$ qui appellent à mieux identifier le type de régime politique dans lequel la répression est mise en œuvre d'une part, la trajectoire des individus impliqués tant du côté de l'État que des collectifs contestataires d'autre part, et enfin l'espace dans lequel leurs interactions se déploient.

Cette démarche n'est cependant pas nouvelle pour les spécialistes de l'engagement militant qui ont redécouvert l'approche interactionniste en Europe à la fin des années 1990, en France notamment. Mettant les histoires de vie au centre de l'analyse, ces derniers se servent des notions de «carrière » ou de «trajectoire » militante pour comprendre l'imbrication entre les histoires individuelles, les institutions et le contexte politique dans lequel elles s'inscrivent ${ }^{6}$.

Malgré leurs différents angles d'approche, les travaux américains et francophones attachés à l'étude de l'action collective partagent un intérêt particulier pour les logiques d'adaptation de la contestation aux dispositifs répressifs. L'exploration des parcours des nouveaux entrants en militantisme s'avère dès lors heuristique pour comprendre l'avènement et l'évolution de dynamiques organisationnelles propres aux contextes répressifs, notamment celles associées à la radicalisation politique ${ }^{7}$.

2. On songe à Lichbach M., Gurr T., "The Conflict Process: A Formal Model”, The Journal of Conflict Resolution, vol. 25, n 1, 1981, pp. 3-29 ; Muller E. N., Weede E., "Cross-National Variation in Political Violence: A Rational Action Approach", Journal of Conflict Resolution, vol. 34, n 4, 1990, pp. 624-651 ; Francisco R., "Coercion and Protest: An Empirical Test in Two Democratic States", American Journal of Political Science, vol. 40, $\mathrm{n}^{\circ}$ 4, 1996, pp. 1179 1204.

3. Voir par exemple Price R. M., The Apartheid State in Crisis. Political Transformation in South Africa, 1975-1990, New York, Oxford University Press, 1990 ; Lichbach M. I., The Rebel's Dilemma, Ann Arbor, University of Michigan Press, 1995.

4. Goldstone J. A., Tilly C., "Threat (and Opportunity): Popular Action and State Response in the Dynamic of Contentious Action", in Aminzade R. et al., Silence and Voice in the Study of Contentious Politics, Cambridge, Cambridge University Press, 2001, pp. 179-194.

5. Davenport C., Johnston H., Mueller C., Repression and Mobilization, Minneapolis, University of Minnesota Press, 2005.

6. Fillieule O., « Post-scriptum : propositions pour une analyse processuelle de l'engagement individuel ", Revue française de science politique, vol. 5, n 1-2, 2001, pp. 199-215. Pour une revue de la littérature sur le militantisme, nous renvoyons à Sawicki F., Siméant J., « Décloisonner la sociologie de l'engagement militant. Note critique sur quelques tendances récentes des travaux français ", Sociologie du travail, vol. 51, n 1, 2009, pp. 97-125.

7. Zwerman G., Steinhoff P., "When Activists Ask for Trouble: State-Dissident Interactions and the New Left Cycle of Resistance in the United States and Japan", in Davenport C., op. cit., pp. 85-107. 
De récentes revues de la littérature sur la violence politique ${ }^{8}$, sur le processus de radicalisation violente ${ }^{9}$ et sur le rapport entre répression et protestation 10 pointent les angles morts de la recherche dans ces domaines et ouvrent de nouvelles pistes de recherche. Ces travaux de synthèse rappellent l'importance du type de sources utilisées pour produire de nouvelles connaissances. Ce faisant, ils invitent à la production de matériaux empiriques de type qualitatif, impliquant des immersions longues sur le terrain et soulignent l'intérêt d'étudier les dynamiques des organisations militantes engagées dans des processus de radicalisation politique, tout spécialement à l'échelle des individus qui les font évoluer. Il s'agit en ce sens aussi bien de rendre compte des formes par lesquelles ces organisations adoptent la violence révolutionnaire que d'expliquer la portée de leurs processus de radicalisation politique et de comprendre comment l'action violente est ensuite abandonnée au cours de processus de déradicalisation.

Axé sur l'étude des pratiques et des savoir-faire militants, ce dossier explore certaines de ces pistes de recherche. Les articles ici proposés exploitent en priorité des données empiriques issues d'enquêtes ethnographiques et socio-biographiques.

Il s'agit tout d'abord de penser le rapport à la répression comme un moyen pour les militants de confirmer leur identité politique et d'être reconnus pour leur hérö̈sme par leurs dirigeants et leurs pairs. Dans cette perspective, le cas du Parti national-bolchevique russe (1990-2000) étudié par Véra Nikolski montre comment la prise de risque face à des niveaux de répression intense se trouve au principe d'une logique de classement sur une échelle de prestige interne à l'organisation, suscitant la fierté chez ceux qui s'exposent le plus au danger. Véra Nikolski opère un renversement de perspective en montrant que la répression est moins subie que recherchée et surtout qu'elle peut devenir un « plaisir ». Le désir de répression est également présent dans le cas du Parti communiste français (PCF) étudié par Vanessa Codaccioni pendant la guerre froide et la guerre d'Algérie (1947-1962). La répression des jeunes militants du PCF représente un réel enjeu stratégique et pédagogique pour l'organisation partisane, car c'est dans le rapport à la répression que les nouvelles recrues incorporent des pratiques ainsi que les règles et les normes partisanes. Dans ce cas, la répression conduit les militants à réinvestir les acquis de leur socialisation politique pour consolider leur appartenance partisane. Cette dynamique les amène parfois à être repérés pour devenir de nouveaux

8. Della Porta D., « Mouvements sociaux et violence politique », in Crettiez X., Mucchielli L. (eds.), Les violences politiques en Europe. Un état des lieux, Paris, La Découverte, 2010, pp. 271-292.

9. Crettiez X., «"High risk activism" : essai sur le processus de radicalisation violente (première partie) », Pôle Sud, n 34, 2011, pp. 45-60.

10. Combes H., Fillieule O., «De la répression considérée dans ses rapports à l'activité protestataire. Modèles structuraux et interactions stratégiques ", Revue française de science politique, vol. 61, n 6, 2011, pp. 1047-1072. 
cadres partisans. Mais si les affrontements avec les agents du maintien de l'ordre renforcent les liens partisans au cours du processus de radicalisation, le processus de déradicalisation qui s'ensuit distend ces liens, générant des flux de défection intenses qui participent à terme au déclin du PCF.

Un deuxième point commun aux textes ici réunis se trouve dans l'intérêt porté à la socialisation politique des militants formés à l'action violence face à la répression et à leurs devenirs dans la période post-répressive. En témoigne l'étude de Daniela Cuadros sur les nouveaux entrants au Parti communiste du Chili (PCCh) dans les années 1983-1986, au sommet de la protestation contre le régime dictatorial dirigé par le général Pinochet. À la différence du PCF des années 1947-1962, il semble qu'au Chili, l'emprise du groupe dirigeant communiste sur le corps militant ait été considérablement affaiblie au cours du processus de radicalisation (1980-1986), de telle sorte que la nouvelle génération militante issue de ce processus se forme dans un rapport distancié à l'organisation partisane. Le devenir du PCCh dans l'étape suivante, celle de la déradicalisation survenue à la suite de la transition démocratique (1987-1989), prend la forme d'une crise des vocations militantes toute particulière qui, contre toute attente, ne mène pas systématiquement au désengagement. L'approche par les trajectoires des militants encore actifs dans les années 2006-2008 montre en ce sens que la déradicalisation suit deux mouvements concomitants, la reconstruction douloureuse de l'attachement partisan d'un côté et le maintien d'un rapport conflictuel des militants avec les agents répressifs dans le nouveau contexte démocratique (1990-2010) de l'autre.

Les contributions se rejoignent également dans l'intérêt porté aux processus de désengagement caractéristiques des périodes post-répressives. Le cas des militants des nouvelles gauches aux États-Unis et au Japon à la fin des années 1960 et dans les années 1970 témoigne du rôle central joué par les réseaux d'aide juridique dans la production de conditions favorables à l'engagement radical suivant trois phases principales : l'entrée dans la lutte armée, la persistance dans la clandestinité et l'abandon de l'action violente. Patricia Steinhoff et Gilda Zwerman dévoilent ainsi les conditions sociales et les situations personnelles qui conduisent à la clandestinité. Ce faisant, elles étudient la manière dont les exigences de la clandestinité ont transformé les militants des douze organisations armées auprès de qui elles ont enquêté pendant plusieurs années. La comparaison dévoile les traits communs des cycles de protestation dans les deux pays, ainsi que l'impact de la répression sur les militants. Enfin, l'article de Guya Accornero propose une analyse des conséquences de la politique répressive de l'Estado Novo (1933-1974) sur les mouvements d'opposition étudiants au Portugal. Il est centré sur l'examen de la trajectoire de José Luís Saldanha Sanches, un militant originellement membre du Parti communiste portugais qui s'est reconverti au maö̈sme durant la répression. 
Les causes censées inspirer les organisations réprimées ne sont donc pas l'objet de ce dossier. Il s'agit plutôt de suivre la piste, à l'échelle des individus, des circonstances répressives ayant amené les organisations militantes à s'adapter, à se transformer et - comme dans la plupart des cas évoqués ici - à survivre à des conditions pour le moins défavorables à la contestation. L'invitation est à découvrir comment et dans quelle mesure les organisations sont susceptibles de s'adapter en se radicalisant face au «danger » répressif (sous des conditions essentiellement différentes) et à observer comment, après la période répressive, les militants sont amenés à se désengager ou à se reconvertir au cours de processus de déradicalisation qu'ils participent à produire. 